\title{
Detection of chromosome abnormalities using current noninvasive prenatal testing: A multi-center comparative study
}

\author{
Yan Du ${ }^{1,2,3, \S}$, Jing Lin ${ }^{1,3,4,}$, Likun Lan ${ }^{5}$, Ying Dong ${ }^{6}$, Jun Zhu ${ }^{7}$, Wen Jiang ${ }^{8}$, Xinyao Pan ${ }^{1,3,4}$, \\ Youhui $\mathrm{Lu}^{1,3,4}$, Dajin $\mathrm{Li}^{1,3,4}$, Ling Wang ${ }^{1,3,4, *}$ \\ ${ }^{1}$ Laboratory for Reproductive Immunology, Hospital \& Institute of Obstetrics and Gynecology, Shanghai Medical College, Fudan \\ University, Shanghai, China; \\ ${ }^{2}$ Office of Clinical Epidemiology, Obstetrics and Gynecology Hospital of Fudan University, Shanghai, China; \\ ${ }^{3}$ The Academy of Integrative Medicine of Fudan University, Shanghai, China; \\ ${ }^{4}$ Shanghai Key Laboratory of Female Reproductive Endocrine-related Diseases, Shanghai, China; \\ ${ }^{5}$ Second Affiliated Hospital of Hexi University, Gansu, China; \\ ${ }^{6}$ Putuo District Institute of Maternity and Child Health of Shanghai, Shanghai, China; \\ ${ }^{7}$ Department of Obstetrics and Gynecology, Wenling People's Hospital, Wenzhou Medical University, Zhejiang, China; \\ ${ }^{8}$ Department of Obstetrics and Gynecology, Zhoushan Maternity and Child Healthcare Hospital, Zhejiang, China.
}

Summary Noninvasive prenatal testing (NIPT) is increasingly recognized and utilized in the antenatal care field. In the current study, we aimed to evaluate the clinical application and compare test outcomes of two generations of currently used NIPT techniques for detecting fetal chromosome abnormalities in a high-risk prenatal population. A total of 7,252 pregnant women were included from twenty-one hospitals from January 2015 to September 2017. A maternal blood sample of each participant was collected for fetal DNA sequencing. Group I received a first generation NIPT sequencing technique to detect chromosome aneuploidies, and Group II received a second generation NIPT sequencing technique to detect subchromosome abnormalities. An abnormal NIPT result was reported in $0.90 \%(44 / 4,868)$ of the women in Group I and 2.68\% (64/2,384) in Group II. In Group I, seventeen (17/37, 45.95\%) women with suspected fetal aneuploidy received amniocentesis, which confirmed $100 \%(10 / 10)$ of positive trisomy 21 samples, $100 \%$ (1/1) of trisomy 18, 100\% (1/1) of sex chromosome abnormality, $0 \%(0 / 2)$ of trisomy $16,0 \%(0 / 2)$ of trisomy 13 , and $0 \%(0 / 1)$ of trisomy 20 and 13 . In Group II, aneuploidy accounted for $46.88 \%(30 / 64)$ of the abnormal results. Five underwent amniocentesis and three had an abnormal result, including two cases of trisomy 21 and one case of chromosome $5 p$ deletion syndrome. Whereas one case of 46,XN,del(16q11.2-q22.3) and another case of $46, \mathrm{XN}, \mathrm{dup}(\mathrm{Xp22}$.31) were considered as normal. NIPT is a quick and reliable screening method for detecting fetal chromosome aneuploidies and subchromosome deletions/ duplications. Challenges remain for the comprehensive clinical application of NIPT.

Keywords: Noninvasive prenatal testing (NIPT), prenatal testing, chromosome aneuploidies, subchromosome deletions/duplications

\section{Introduction}

Prenatal testing is designed to acquire a fetal biological

Released online in J-STAGE as advance publication June 28, 2018.

${ }^{\S}$ These authors contributed equally to this work.

*Address correspondence to:

Dr. Ling Wang, Laboratory for Reproductive Immunology, Hospital \& Institute of Obstetrics and Gynecology, Fudan University, 419 Fangxie Road, Shanghai 200011, China.

E-mail: Dr.wangling@fudan.edu.cn and genetic profile and thus detect any potential genetic abnormalities of the fetus. Prenatal screening for fetal health assessment involves maternal serum biochemical markers and ultrasound scan, which have limitations such as the need to combine a series of markers or to be performed at different time points. Prenatal diagnosis, including amniocentesis and chorionic villus sampling, provides information about the fetal karyotype at a certain gestational window and has its clinical applicability; however, it can also cause risks for the fetus (1). With the advancement of highly accurate 
noninvasive approaches, new options for prenatal testing become available.

Noninvasive prenatal testing (NIPT) which analyzes cell-free DNA (cfDNA) of fetal origin in maternal circulation for detection of fetal chromosome abnormalities in high-risk pregnancies has received widespread recognition and utilization since 2011 (2). NIPT offers high sensitivity and specificity for common fetal chromosome aneuploidies such as trisomy 21, 18 , and 13. It has been proposed that the indication of NIPT can be expanded to all autosomes and even subchromosome deletions/duplications (3). It has also been recommended to incorporate cfDNA screening into current clinical prenatal practice as a screening alternative for various genetic conditions. Moreover, guidelines of the American College of Medical Genetics and Genomics (ACMG) have suggested that NIPT may replace conventional screening of common aneuploidies for women across the maternal age spectrum (4).

NIPT marks a revolution in prenatal screening and opens up new possibilities, but it has certain limitations, including coverage of only the most common trisomies, the possibility of both false-positive and false-negative results, and occurrence of test failure (5). Despite the above mentioned limitations, NIPT is likely to be increasingly adopted as part of prenatal practice. So far, there are two generations of sequencing techniques of NIPT. The first generation NIPT sequencing mainly detects chromosome aneuploidies, while the second generation NIPT sequencing can also detect subchromosome abnormalities. The most important differences between the first and second generation NIPT sequencing techniques are the length of sequencing reads and depth of coverage. The first generation NIPT sequencing technique for chromosome aneuploidies is based on reads up to $1,000 \mathrm{bp}$, while the size of reads for the second generation NIPT sequencing technique is up to $400 \mathrm{bp}$. Therefore, we investigated the clinical application and compared the outcomes of the two NIPT techniques for direct detection of fetal chromosome aneuploidies or subchromosome deletions/ duplications in women with high-risk pregnancies.

\section{Materials and Methods}

\subsection{Participant recruitment}

A total of 7,252 pregnant women were included from January 13th, 2015 to September 30th, 2017 from the following twenty-one hospitals: Zhoushan Maternity and Child Healthcare Hospital, International Peace Maternity and Child Health Hospital, Renji Hospital of Shanghai Jiaotong University School of Medicine, Obstetrics and Gynecology Hospital of Fudan University, Shanghai First Maternity and Infant Hospital, Shanghai First People's Hospital, Shanghai Sixth People's Hospital, Shanghai Eighth People's
Hospital, Shanghai Ninth People's Hospital, Changning Maternity and Infant Health Hospital, Putuo Maternity and Infant Health Hospital, Jiading Maternity and Infant Health Hospital, Hongkou Maternity and Infant Health Hospital, Maternity and Infant Health Hospital of Pudong New District, Shanghai Pudong Hospital, Shanghai Institute of Planned Parenthood Research Hospital, Zhongshan Hospital, Yueyang Hospital, Shuguang Hospital, Minhang District Central Hospital, and Jinshan Hospital. Maternal characteristics that were important risk indicators of fetal chromosome aneuploidy included advanced maternal age, personal history of abnormal gestation and birth, family history of chromosome aneuploidy, positive serum marker screening, and abnormal fetal ultrasound findings. The participants received either the first generation (Group I) or the second generation (Group II) NIPT sequencing technique. Peripheral venous blood of each participating pregnant woman was collected. The study protocol was approved by the institutional review board of Zhoushan Maternity and Child Healthcare Hospital. Informed consent was obtained from all participants.

\section{2. $f D N A$ preparation and sequencing}

Five to $10 \mathrm{~mL}$ of the maternal blood sample was collected and the plasma was separated from peripheral blood cells. Using the QIAamp DSP DNA Blood Mini Kit (Qiagen), cfDNA from $600 \mu \mathrm{L}$ of maternal plasma was extracted following the manufacturer's protocol. DNA fragments were obtained with the NEBNext dsDNA Fragmentase (New England Biolabs). Fetal Chromosome Aneuploid (T21, T18, and T13) Detection Kit (CapitalBio Corporation) was utilized for library construction, library quality control, and library amplification following the manufacturer's protocol. DNA sequencing was performed using the BioelectronSeq 4000 Semiconductor Sequencing System (CapitalBio Corporation) according to the manufacturer's instructions.

\subsection{Statistical analysis}

Age was compared using Student's $t$ test as a continuous variable between the two Groups. The obtained reads were aligned to the human genomic reference sequences (hg19) using the BWA algorithm. Unmapped reads or those with multiple primary alignment records were filtered by FLAG field in the alignment file with an in-house Perl script. An integrated three-step process [LOESS regression (6), intrarun normalization (7), and linear model regression (8)] was applied to eliminate the effect of GC bias. A Z score was calculated to identify fetuses with trisomy $21,18,13$, or sex chromosome aneuploidies. A cutoff value of $\mathrm{Z}$ score $>3$ was set to determine whether the ratio of chromosome 21,18 , or 13 was increased and hence fetal trisomy 21,18 , or 13 was 
present. Then the $\mathrm{Z}$ scores for each $1 \mathrm{Mb}$ region were combined and a Stouffer's Z-score method was adopted to improve the accuracy for detection of subchromosome abnormalities. A Stouffer's Z score $>5$ was determined as microduplication, whereas a Stouffer's Z score $<-5$ was classified as microdeletion.

\section{Results}

\subsection{Study participants}

A total of 7,252 pregnant women were included in this study and received either the first generation (Group I) or the second generation (Group II) NIPT sequencing technique. Group I contained 4,868 pregnant women, using the first generation NIPT sequencing technique to detect chromosome aneuploidies. Group II included 2,384 women, using the second generation NIPT sequencing technique to detect subchromosome abnormalities. The percentage of pregnant women receiving second generation NIPT sequencing technique (Group II) increased significantly from the year 2015 to 2017 (Figure 1).

\subsection{Detection of fetal aneuploidies in Group I}

An abnormal NIPT result (aneuploidy detected or "unclassified" result) was reported in $0.90 \%(44 / 4,868)$ of the women in Group I. Table 1 presents detailed information of abnormal NIPT results in Group I.

We detected fifteen candidate samples with trisomy $21(15 / 44,34.09 \%)$, five with trisomy $13(5 / 44,11.36 \%)$, three with trisomy $20(3 / 44,6.81 \%)$, three with trisomy $18(3 / 44,6.81 \%)$, three with trisomy $16(1 / 44,6.81 \%)$, one with trisomy $15(3 / 44,2.27 \%)$, one with trisomy 9 $(1 / 44,2.27 \%)$, one with trisomy $7(1 / 44,2.27 \%)$, one with trisomy 20 and $13(1 / 44,2.27 \%)$, two with 45,X (2/44, 4.55\%), one with 47,XXX (1/44, 2.27\%), one with unclassified sex chromosome abnormality (1/44, 2.27\%), four samples with microdeletion $(4 / 44,9.09 \%)$, and three with microduplication $(3 / 44,6.82 \%)$. Seventeen $(17 / 37,45.95 \%)$ women with suspected fetal aneuploidy received amniocentesis, which confirmed 100\% (10/10) of positive trisomy 21 samples, $100 \%(1 / 1)$ of trisomy $18,100 \%(1 / 1)$ of sex chromosome abnormality, $0 \%(0 / 2)$ of trisomy $16,0 \%(0 / 2)$ of trisomy 13 , and $0 \%(0 / 1)$ of trisomy 20 and 13 .

\subsection{Detection of fetal subchromosome abnormalities in Group II}

Sixty-four of the $2,384(64 / 2,384,2.68 \%)$ women in Group II who received second generation NIPT sequencing technique to detect subchromosome abnormalities were screen-positive.

Table 2 presents detailed information of abnormal NIPT results in Group II. Aneuploidy accounted for

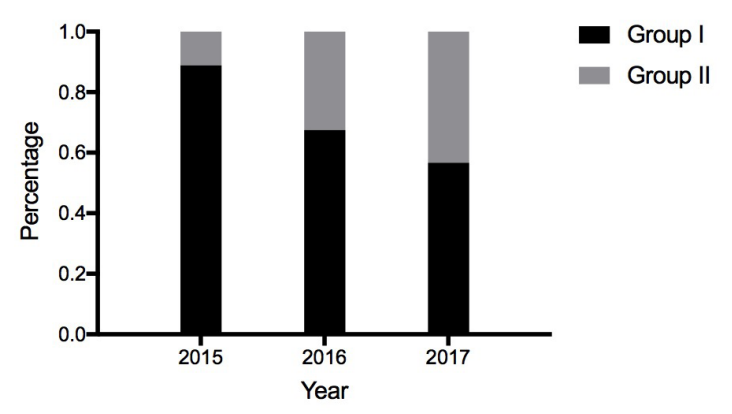

Figure 1. Percentages of pregnant women receiving first (Group I) and second (Group II) generation NIPT sequencing techniques each year, 2015-2017.

Table 1. Detailed information of abnormal NIPT results in Group I

\begin{tabular}{|c|c|c|}
\hline Abnormal NIPT results & $\mathrm{N}$ & $\begin{array}{l}\% \text { of patients with } \\
\text { abnormal results }\end{array}$ \\
\hline \multicolumn{3}{|l|}{ Aneuploidy } \\
\hline $47, \mathrm{XN},+7$ & 1 & $2.27 \%$ \\
\hline $47, \mathrm{XN},+9$ & 1 & $2.27 \%$ \\
\hline $47, \mathrm{XN},+13$ & 5 & $11.36 \%$ \\
\hline $47, \mathrm{XN},+15$ & 1 & $2.27 \%$ \\
\hline $47, \mathrm{XN},+16$ & 3 & $6.82 \%$ \\
\hline $47, \mathrm{XN},+18$ & 3 & $6.82 \%$ \\
\hline $47, \mathrm{XN},+20$ & 3 & $6.82 \%$ \\
\hline $47, \mathrm{XN},+21$ & 15 & $34.09 \%$ \\
\hline $48, \mathrm{XN},+13,+20$ & 1 & $2.27 \%$ \\
\hline $45, \mathrm{X}$ & 2 & $4.55 \%$ \\
\hline $47, \mathrm{XXX}$ & 1 & $2.27 \%$ \\
\hline Unclassified sex chromosome abnormality & 1 & $2.27 \%$ \\
\hline \multicolumn{3}{|l|}{ Deletion } \\
\hline $46, \mathrm{XN}, \operatorname{del}(3)$ & 1 & $2.27 \%$ \\
\hline $46, \mathrm{XN}, \operatorname{del}(4 \mathrm{q} 34.1-\mathrm{q} 35.1)$ & 1 & $2.27 \%$ \\
\hline $46, \mathrm{XN}, \operatorname{del}(10)$ & 1 & $2.27 \%$ \\
\hline 46,XN,del(20) & 1 & $2.27 \%$ \\
\hline \multicolumn{3}{|l|}{ Duplication } \\
\hline 46,XN,dup(3q26.1-q29) & 1 & $2.27 \%$ \\
\hline $46, \mathrm{XN}, \operatorname{dup}(7)$ & 1 & $2.27 \%$ \\
\hline 46,XN,dup(15) & 1 & $2.27 \%$ \\
\hline
\end{tabular}

NIPT, noninvasive prenatal testing.

$46.88 \%(30 / 64)$ of the abnormal results, followed by twenty-five cases of subchromosome duplication (25/64, $39.06 \%$ ), seven cases of subchromosome deletion $(7 / 64,10.94 \%)$, and two cases of both duplication and deletion $(2 / 64,3.13 \%)$. Of all screen-positive women in Group II, five $(5 / 64,7.81 \%)$ underwent amniocentesis and three of them had abnormal results, including two cases of trisomy 21 and one case of chromosome $5 p$ deletion syndrome (Cri-du-chat syndrome). However, one case of 46,XN,del(16q11.2-q22.3) and another case of $46, \mathrm{XN}, \operatorname{dup}(\mathrm{Xp} 22.31)$ were considered as normal according to the results of amniocentesis.

\subsection{Comparison between Group I and Group II}

The average maternal age of Group I was 32 years (range: 17-54 years), and the average gestational age 
Table 2. Detailed information of abnormal NIPT results in Group II

\begin{tabular}{|c|c|c|}
\hline Abnormal NIPT results & $\mathrm{N}$ & $\begin{array}{l}\% \text { of patients with } \\
\text { abnormal results }\end{array}$ \\
\hline \multicolumn{3}{|l|}{ Aneuploidy } \\
\hline $47, \mathrm{XN},+10$ & 1 & $1.56 \%$ \\
\hline $47, \mathrm{XN},+11$ & 2 & $3.13 \%$ \\
\hline $47, \mathrm{XN},+13$ & 5 & $7.81 \%$ \\
\hline $47, \mathrm{XN},+14$ & 1 & $1.56 \%$ \\
\hline $47, \mathrm{XN},+16$ & 1 & $1.56 \%$ \\
\hline $47, \mathrm{XN},+18$ & 3 & $4.69 \%$ \\
\hline $47, \mathrm{XN},+21$ & 13 & $20.31 \%$ \\
\hline $48, \mathrm{XXY},+8$ & 1 & $1.56 \%$ \\
\hline $45, \mathrm{X}$ & 3 & $4.69 \%$ \\
\hline \multicolumn{3}{|l|}{ Deletion } \\
\hline 46,XN,del(5p15.31-15.2) & 1 & $1.56 \%$ \\
\hline $46, \mathrm{XN}, \operatorname{del}(7 \mathrm{q} 21.3-\mathrm{q} 31.2)$ & 1 & $1.56 \%$ \\
\hline $46, \mathrm{XN}, \operatorname{del}(15 \mathrm{q} 21.3)$ & 1 & $1.56 \%$ \\
\hline 46,XN,del(16q11.2-q22.3) & 1 & $1.56 \%$ \\
\hline $46, \mathrm{XN}, \operatorname{del}(17 \mathrm{p} 12)$ & 2 & $3.13 \%$ \\
\hline 46,XN,del(18p11.32) & 1 & $1.56 \%$ \\
\hline \multicolumn{3}{|l|}{ Duplication } \\
\hline 46,XN,dup(1q44) & 1 & $1.56 \%$ \\
\hline $46, X N, \operatorname{dup}(2 q 13)$ & 1 & $1.56 \%$ \\
\hline $46, X N, \operatorname{dup}(2 q 22.3-q 23.1)$ & 1 & $1.56 \%$ \\
\hline 46,XN,dup(2q37.1-q37.2) & 1 & $1.56 \%$ \\
\hline $46, \mathrm{XN}, \operatorname{dup}(2 \mathrm{p} 24.3)$ & 1 & $1.56 \%$ \\
\hline 46,XN,dup(4q21.1-q21.21) & 1 & $1.56 \%$ \\
\hline $46, \mathrm{XN}, \operatorname{dup}(4 \mathrm{q} 35.2)$ & 1 & $1.56 \%$ \\
\hline $46, X N, \operatorname{dup}(5 q 14.3)$ & 1 & $1.56 \%$ \\
\hline $46, \mathrm{XN}, \operatorname{dup}(6 \mathrm{p} 12.1)$ & 1 & $1.56 \%$ \\
\hline 46,XN,dup(7q11.22-q11.23) & 1 & $1.56 \%$ \\
\hline $46, \mathrm{XN}, \operatorname{dup}(10 \mathrm{p} 14)$ & 1 & $1.56 \%$ \\
\hline $46, \mathrm{XN}, \operatorname{dup}(12 \mathrm{q} 21.1)$ & 1 & $1.56 \%$ \\
\hline $46, \mathrm{XN}, \operatorname{dup}(14 \mathrm{q} 31.3)$ & 1 & $1.56 \%$ \\
\hline $46, X N, \operatorname{dup}(16 q 23.3-q 24.1)$ & 1 & $1.56 \%$ \\
\hline $46, \mathrm{XN}, \operatorname{dup}(17 \mathrm{q} 12)$ & 1 & $1.56 \%$ \\
\hline 46,XN,dup(20p12.1) & 1 & $1.56 \%$ \\
\hline $46, X N, \operatorname{dup}(22 q 11.23)$ & 2 & $3.13 \%$ \\
\hline 46,XN,dup(Xp21.3) & 1 & $1.56 \%$ \\
\hline 46,XN,dup(Xp22.31) & 6 & $9.38 \%$ \\
\hline \multicolumn{3}{|l|}{ Deletion and duplication } \\
\hline 46,XN,dup(1q41-q44), del(13q32.3-q34) & 1 & $1.56 \%$ \\
\hline 46,XN,dup(7p21.3), del(19q13.2-q13.31) & 1 & $1.56 \%$ \\
\hline
\end{tabular}

NIPT, noninvasive prenatal testing.

was $17^{+1}$ weeks (range: $6^{+0}-36^{+6}$ weeks). The mean maternal age of Group II was 33 years (range: 1247 years), with a corresponding gestational age of $15^{+3}$ weeks (range $4^{+0}-34^{+6}$ weeks). There was no age difference $(P=0.20)$ between the two groups. However, the gestational age was significantly earlier in Group II compared to that in Group I $(P<0.001)$. NIPT was most actively selected at the 13th and the 9th weeks of gestation in Group I and II, respectively. The peak age range to receive NIPT in both groups was around 27-30 and 34-37 years, respectively. Figure 2A and 2B present the distribution of age and gestational week of the two groups.

The average maternal age was 33.2 years and the average gestational age was $16^{+5}$ weeks among 44 NIPT-positive women in Group I. The average maternal age and average gestational age of 64 NIPT-positive women in Group II was 33.8 years and $14^{+4}$ weeks, respectively. There was no age difference $(P=0.64)$ of NIPT-positive women between the two groups. However, the gestational age was significantly earlier for NIPT-positive women in Group II compared to that in Group I $(P<0.007)$. Figure 3A and 3B show the distribution of maternal age and gestational age among NIPT-positive women in Group I and Group II, respectively.

Figure 4 shows the NIPT process and results.

\section{Discussion}

The rate of abnormal NIPT result was $0.90 \%(44 / 4,868)$ in Group I and 2.68\% $(64 / 2,384)$ in Group II. Our results showed that aneuploidy accounted for $84.09 \%$ (37/44) of the abnormal results in Group I, and 46.88\% (30/64) in Group II. Notably, subchromosome deletions/duplications comprised a higher percentage of abnormal NITP results in Group II (34/64, 53.13\%) compared to Group I (7/44, 15.91\%), which could be explained by the fact that the second generation sequencing technique offered a higher throughput and more sensitive platform to generate accurate data and unexpected DNA variation, thus increasing the screenpositive rate and the detection rate of subchromosome abnormalities. The reported screen-positive rate varied according to the technique selected and population studied. The Harmony test conducted in Belgium and the Netherlands reported a screen-positive rate of $1.9 \%$ $(57 / 3,000)$ of pregnancies $(9)$.

The performance of the laboratory developed NIPT technology was evaluated using fetal karyotype results obtained from invasive approaches in singleton pregnancies as the gold standard. Of screen-positive women in Group I, 38.64\% (17/44) received invasive diagnostic testing and $70.59 \%(12 / 17)$ of those tested had an abnormal result. Amniocentesis confirmed ten positive trisomy 21 samples, one positive trisomy 18 , and one positive sex chromosome abnormality; while two cases of trisomy 16 , two cases of trisomy 13 , and one case of trisomy 20 and 13 were considered as normal. In Group II, five (5/64, 7.81\%) women underwent amniocentesis and three had an abnormal result. Two cases of trisomy 21 and one case of Cridu-chat syndrome were confirmed by amniocentesis, whereas one case of 46,XN,del(16q11.2-q22.3) and another case of $46, \mathrm{XN}$,dup(Xp22.31) were considered as normal. Previous studies have reported that NIPT could be successfully validated for common aneuploidies in singleton pregnancies; and the detection rates were $99.7 \%$ for trisomy $21,98 \%$ for trisomy 18 , and $99 \%$ for trisomy 13 with a combined false-positive rate (FPR) of $0.13 \%(10)$, and the detection rate for subchromosome abnormalities was $71.8 \%$ (11). Another study using NIPT to identify common aneuploidies reported that the sensitivity and specificity were 

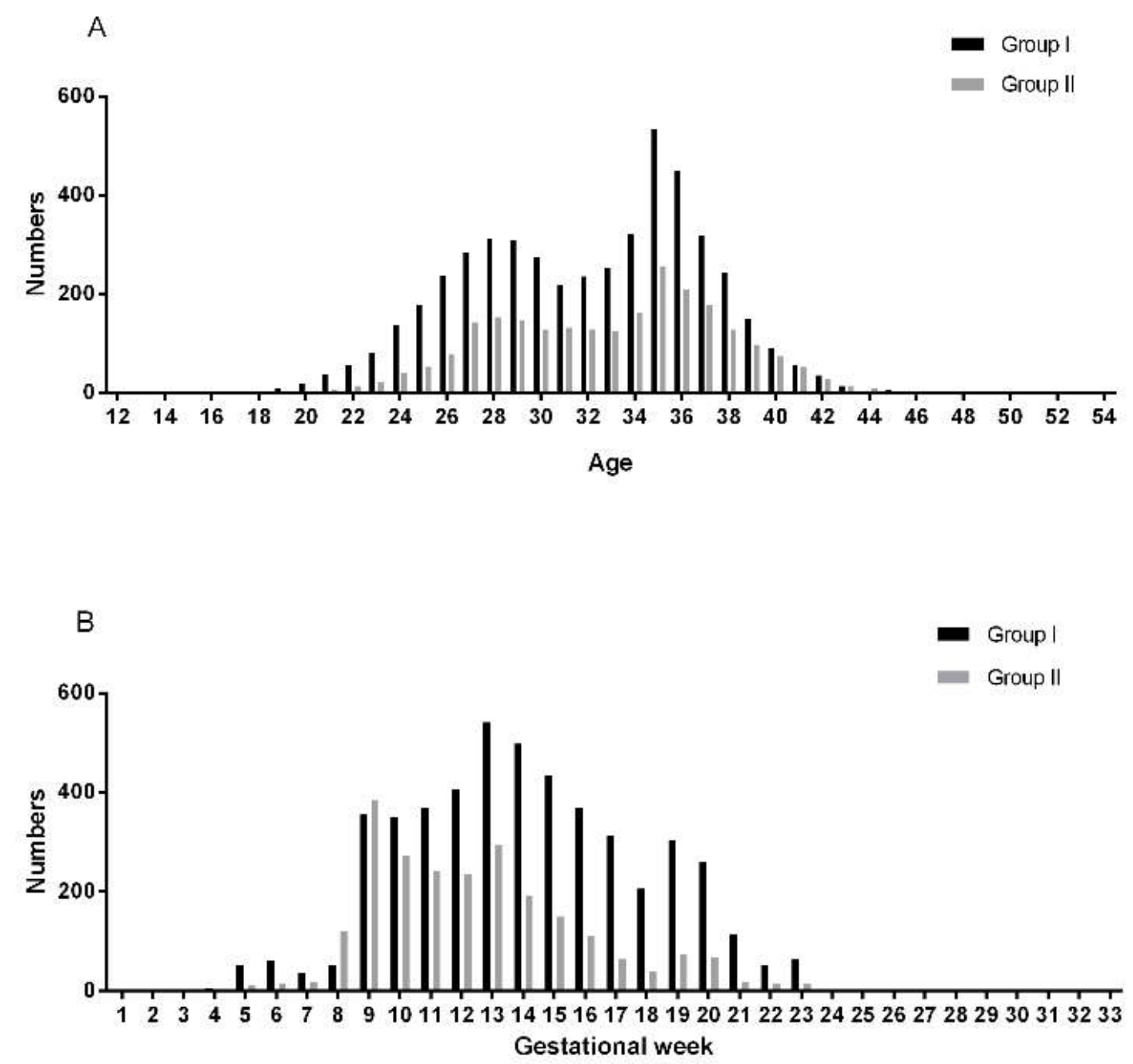

Figure 2. Distribution of maternal age and gestational week of all participants in Group I $(n=4,868)$ and Group II $(n=$ 2,384). A: age; B: gestational week.
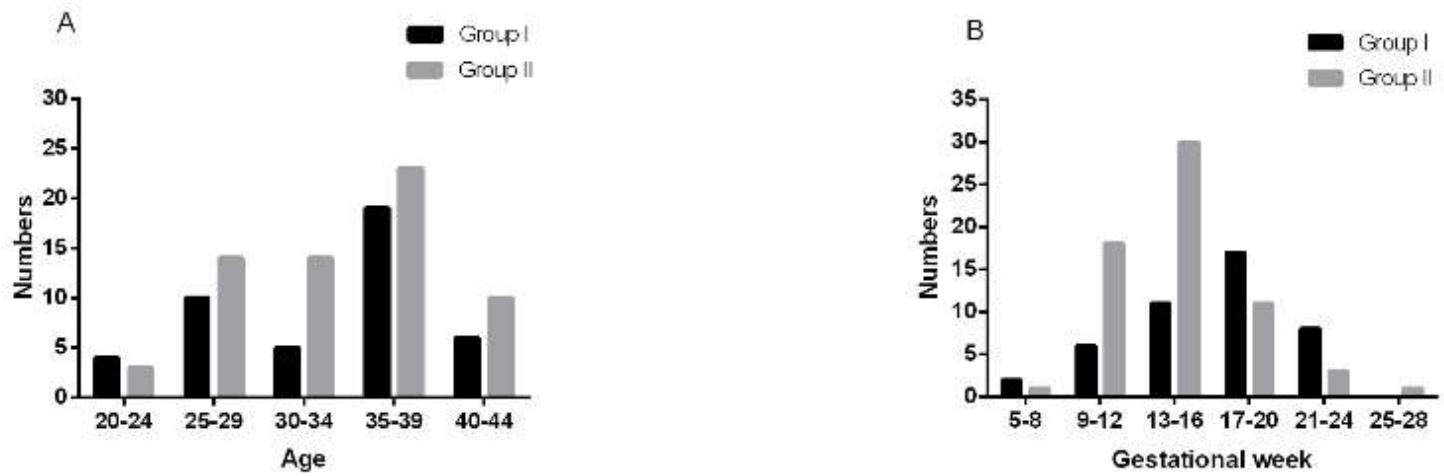

Figure 3. Distribution of NIPT-positive women by maternal age and gestational age in Group I $(n=44)$ and Group II $(n=64)$. A: age; B: gestational week.

$99.94 \%$ and $99.46 \%$ for trisomy $21,100 \%$ and $99.24 \%$ for trisomy 18 , and $100 \%$ and $100 \%$ for trisomy 13 , respectively (12). Data from a large referral genetic diagnostic laboratory in the United States showed that the positive predictive values (PPV) were $84 \%$ for trisomy $21,76 \%$ for trisomy $18,45 \%$ for $13,50 \%$ for trisomy X, 26\% for monosomy X, and $0 \%$ to $21 \%$ for microdeletion syndromes (13). In line with the above findings, our results also demonstrated that NIPT has better performance when screening for trisomy 21 than other aneuploidies, while the FPR is higher and the PPV is lower when NIPT is used to screen for 


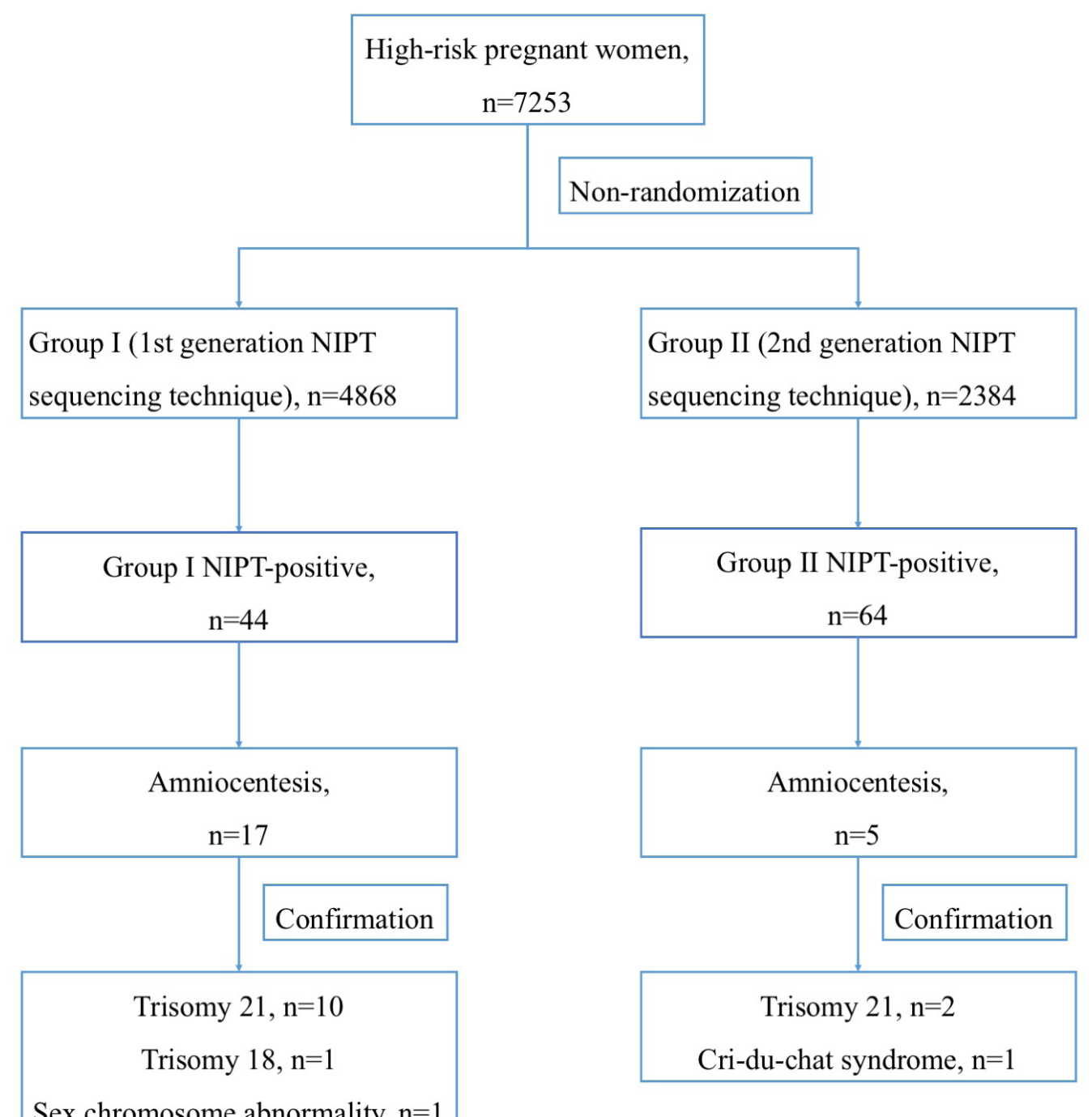

Figure 4. Flow chart of NIPT process and results.

subchromosome abnormalities.

Compared with traditional screening methods and invasive approaches, cfDNA-based NIPT detects the core pathology of chromosome abnormalities instead of epiphenomenon. In addition, NIPT also has minimal risks of fetal losses compared to invasive procedures. It is estimated that the implementation of NIPT as a second step screening procedure in high-risk pregnancies would lead to a $10.2 \%$ increase in detected Down syndrome cases and a $96.3 \%$ decrease in fetal losses (14). However, NIPT needs to be implemented in combination with other prenatal tests to avoid certain limitations.

NIPT is selected mainly for reassurance by low-risk pregnant women in the clinical setting (15). Studies have shown that introduction of NIPT as a second-tier screen was not associated with a decrease of invasive testing in the high-risk population (16). Furthermore, NIPT could detect unconfirmed results or variants of unknown clinical significance, ranging from relatively healthy newborns to those associated with fetal abnormality or pregnancy complication (17). With the increasing utilization of the second generation NIPT sequencing technique as seen in our study, how to decrease FPR and increase PPV becomes an important issue to avoid unnecessary stress for pregnant women.

From the biological perspective, fetoplacental mosaicism is one of the underlying causes of falsepositives and false-negatives in NIPT, since cfDNA originates from the mother and the placenta (trophoblasts) rather than from the fetus proper (18). Other causes of false positive or negative results include: pregnancy with twins or triplets; women with chromosome disorders, malignancy, or previous treatments such as transplant, stem-cell treatment, immune therapy, blood transfusion, and in vitro fertilization-embryo transfer; the interference of fetal balanced translocation, mosaic trisomy; and inappropriate collection, transferring, and handling of the samples (5). Meanwhile, studies have shown that approximately $15 \%$ of chromosome abnormalities 
would not be identified by NIPT among those highrisk women with abnormalities who pursued prenatal diagnostic testing (10). Moreover, NIPT cannot be used to screen for the broad term of "any genetic condition", such as cleft palette, deafness, neural tube defects, or autism. Recently some researchers have suggested expanding the indication of NIPT to all autosomes and even subchromosome deletion/duplication syndromes (3). However, the majority of published consensus or recommendations from the professional societies are not in favor of the clinical implementation of NIPT to detect fetal microdeletions (19).

On the technological side, there are several features of the sequencing platform that restrict the extensive application of NIPT, which include high test price, requirement of fetal fraction above the threshold, test failure after repeated sampling, difficulties to detect small aberrations, and high false positive rate (20). Moreover, standard infrastructure, innovative algorithm, as well as personnel expertise are required for subsequent data analysis (21).

In conclusion, NIPT is increasingly recognized and accepted in the antenatal care field, because of the cost reduction and repertoire expansion to add subchromosome abnormalities besides the originally included trisomy $21,18,13$, and sex chromosome aneuploidies. With the expanding availability of NIPT, health providers are challenged to educate patients about the benefits, limitations, costs, risks, results, and follow-up options of NIPT for adequate informed consent. Our study provided further evidence to support the utilizations and benefits of the cell-free fetal DNA screening technology. It is crucial to improve the laboratory standards for testing and reporting, execute pre- and post-test counseling, identify clearer indicators for invasive testing, and perform continuous evaluation in order to comprehensively establish the clinical application of NIPT.

\section{Acknowledgements}

This work was supported by the National Natural Science Foundation of China No. 31571196 (to L Wang), the Science and Technology Commission of Shanghai Municipality 2015 YIXUEYINGDAO project No. 15401932200 (to L Wang), the FY2008 JSPS Postdoctoral Fellowship for Foreign Researchers P08471 (to L Wang), the National Natural Science Foundation of China No. 30801502 (to L Wang), the Shanghai Pujiang Program No. 11PJ1401900 (to L Wang), Development Project of Shanghai Peak Disciplines-Integrative Medicine No. 20150407.

\section{References}

1. Wilson RD, Gagnon A, Audibert F, Campagnolo C, Carroll J. Prenatal diagnosis procedures and techniques to obtain a diagnostic fetal specimen or tissue: Maternal and fetal risks and benefits. J Obstet Gynaecol Can. 2015; 37:656-668.

2. Benn P, Borrell A, Cuckle H, Dugoff L, Gross S, Johnson JA, Maymon R, Odibo A, Schielen P, Spencer K, Wright D, Yaron Y. Prenatal detection of Down syndrome using massively parallel sequencing (MPS): A rapid response statement from a committee on behalf of the Board of the International Society for Prenatal Diagnosis, 24 October 2011. Prenat Diagn. 2012; 32:1-2.

3. Helgeson J, Wardrop J, Boomer T, Almasri E, Paxton WB, Saldivar JS, Dharajiya N, Monroe TJ, Farkas DH, Grosu DS, McCullough RM. Clinical outcome of subchromosomal events detected by whole-genome noninvasive prenatal testing. Prenat Diagn. 2015; 35:9991004.

4. Gregg AR, Skotko BG, Benkendorf JL, Monaghan KG, Bajaj K, Best RG, Klugman S, Watson MS. Noninvasive prenatal screening for fetal aneuploidy, 2016 update: A position statement of the American College of Medical Genetics and Genomics. Genet Med. 2016; 18:10561065.

5. Harraway J. Non-invasive prenatal testing. Aust Fam Physician. 2017; 46:735-739.

6. Alkan C, Kidd JM, Marques-Bonet T, Aksay G, Antonacci F, Hormozdiari F, Kitzman JO, Baker C, Malig M, Mutlu O, Sahinalp SC, Gibbs RA, Eichler EE. Personalized copy number and segmental duplication maps using next-generation sequencing. Nat Genet. 2009; 41:1061-1067.

7. Jiang F, Ren J, Chen F, et al. Noninvasive Fetal Trisomy (NIFTY) test: An advanced noninvasive prenatal diagnosis methodology for fetal autosomal and sex chromosomal aneuploidies. BMC Med Genomics. 2012; 5:57.

8. Chen EZ, Chiu RW, Sun H, et al. Noninvasive prenatal diagnosis of fetal trisomy 18 and trisomy 13 by maternal plasma DNA sequencing. PLoS One. 2011; 6:e21791.

9. Willems PJ, Dierickx H, Vandenakker E, Bekedam D, Segers N, Deboulle K, Vereecken A. The first 3,000 noninvasive prenatal tests (NIPT) with the Harmony test in Belgium and the Netherlands. Facts Views Vis Obgyn. 2014; 6:7-12.

10. Gil MM, Accurti V, Santacruz B, Plana MN, Nicolaides KH. Analysis of cell-free DNA in maternal blood in screening for aneuploidies: Updated meta-analysis. Ultrasound Obstet Gynecol. 2017; 50:302-314.

11. Yin AH, Peng CF, Zhao X, et al. Noninvasive detection of fetal subchromosomal abnormalities by semiconductor sequencing of maternal plasma DNA. Proc Natl Acad Sci U S A. 2015; 112:14670-14675.

12. Liao C, Yin AH, Peng CF, et al. Noninvasive prenatal diagnosis of common aneuploidies by semiconductor sequencing. Proc Natl Acad Sci U S A. 2014; 111:74157420.

13. Petersen AK, Cheung SW, Smith JL, Bi W, Ward PA, Peacock S, Braxton A, Van Den Veyver IB, Breman AM. Positive predictive value estimates for cell-free noninvasive prenatal screening from data of a large referral genetic diagnostic laboratory. Am J Obstet Gynecol. 2017; 217:691.

14. Okem ZG, Orgul G, Kasnakoglu BT, Cakar M, Beksac MS. Economic analysis of prenatal screening strategies for Down syndrome in singleton pregnancies in Turkey. Eur J Obstet Gynecol Reprod Biol. 2017; 219:40-44. 
15. Manegold-Brauer G, Berg C, Flock A, Ruland A, Gembruch U, Geipel A. Uptake of non-invasive prenatal testing (NIPT) and impact on invasive procedures in a tertiary referral center. Arch Gynecol Obstet. 2015; 292:543-548.

16. Maxwell S, Dickinson JE, Murch A, O'Leary P. The potential impact of NIPT as a second-tier screen on the outcomes of high-risk pregnancies with rare chromosomal abnormalities. Aust N Z J Obstet Gynaecol. 2015; 55:420426.

17. Neofytou MC, Tsangaras K, Kypri E, Loizides C, Ioannides M, Achilleos A, Mina P, Keravnou A, Sismani C, Koumbaris G, Patsalis PC. Targeted capture enrichment assay for non-invasive prenatal testing of large and small size sub-chromosomal deletions and duplications. PLoS One. 2017; 12:e171319.
18. Taglauer ES, Wilkins-Haug L, Bianchi DW. Review: Cellfree fetal DNA in the maternal circulation as an indication of placental health and disease. Placenta. 2014; 35 Suppl:S64-S68.

19. ACOG. Committee opinion No. 640: Cell-free DNA screening for fetal aneuploidy. Obstet Gynecol. 2015.

20. Liu L, Li K, Fu X, Chung C, Zhang K. A forward look at noninvasive prenatal testing. Trends Mol Med. 2016; 22:958-968

21. Allyse M, Minear MA, Berson E, Sridhar S, Rote M, Hung A, Chandrasekharan S. Non-invasive prenatal testing: A review of international implementation and challenges. Int J Womens Health. 2015; 7:113-126.

(Received March 19, 2018; Revised May 31, 2018; Accepted June 10, 2018) 